\title{
Towards a framework approach to integrating pathways for infection prevention and antibiotic stewardship in surgery: a qualitative study from India and South Africa
}

\section{Sanjeev Singh}

Amrita Institute of Medical Sciences

Marc Mendelson

University of Cape Town

Surya Surendran

Amrita Institute of Medical Sciences

Candice Bonaconsa

University of Cape Town

Oluchi Mbamalu

University of Cape Town

\section{Vrinda Nampoothiri}

Amrita Institute of Medical Sciences

Adam Boutall

University of Cape Town

Mark Hampton

University of Cape Town

\section{Puneet Dhar}

Amrita Institute of Medical Sciences

\section{Tim Pennel}

University of Cape Town

Carolyn Tarrant

University of Leicester

Andy Leather

King's College London

Alison Holmes

Imperial College London

Esmita Charani ( $\sim$ e.charani@imperial.ac.uk)

Imperial College London https://orcid.org/0000-0002-5938-1202 
Research article

Keywords: antibiotic prescribing, infection control, ethnography, low- and middle-income country, surgery

Posted Date: September 21st, 2020

DOI: https://doi.org/10.21203/rs.3.rs-76635/v1

License: (c) (i) This work is licensed under a Creative Commons Attribution 4.0 International License.

Read Full License 


\section{Abstract \\ Background}

The surgical pathway remains a hard to reach, critical target for antimicrobial stewardship (AMS) and infection prevention and control (IPC). We investigated the drivers for surgical AMS and IPC, across cardiovascular and thoracic surgery (CVTS) and gastrointestinal surgery teams in two academic hospitals in South Africa (SA) and India.

\section{Materials and methods}

An ethnographic observational study of IPC and AMS was conducted (July 2018-August 2019), with data gathered from 190 hours of non-participant observations (138 India, 60 SA); face-to-face interviews with patients (6 India, 7 South Africa), and healthcare professionals (HCPs) (44 India, 61 SA); and, indepth patient case studies (4 India, 2 SA). A grounded theory approach aided by Nvivo 11 software, analyzed the emerging themes. An iterative and recursive process of moving between the coded data and the higher-level themes, ensured saturation of the themes. The multiple modes of enquiry enabled crossvalidation and triangulation of findings.

\section{Results}

Across surgical pathways, multiple barriers exist impeding effective IPC and AMS practices. The existing, implicit roles of HCPs (including nurses, and senior surgeons) are overlooked as interventions target junior doctors, bypassing the opportunity for integrating care across the surgical team members. Critically, the ownership of decisions remains with the operating surgeons and entrenched hierarchies restrict the integration of other HCPs in IPC and AMS.

\section{Conclusions}

IPC and AMS are not integrated in surgery. Identifying the implicit existing HCPs roles in IPC and AMS is critical and will facilitate the development of effective and transparent processes across the surgical team for IPC and AMS. Developing a framework approach that includes nurse leadership, empowering pharmacists and engaging surgical leads is essential for integrated care.

\section{Background}

Delivering safe surgical care, across the multidisciplinary and multi-locational (spanning the boundaries of primary and secondary care) peri-operative pathways remains a challenge, particularly in low- and middle- income countries (LMICs) ${ }^{1}$. Infections are the most common post-operative complication, with 
LMICs patients more likely to be infected with antibiotic resistant bacteria ${ }^{2}$ and twice as likely to die after an infection-related complication compared to those treated in high-income countries ${ }^{3}$. Surgical site infections, though preventable, are the most common healthcare associated infection ${ }^{4}$. In LMICs, healthcare associated infections remain a significant yet poorly quantified burden, compounded by a lack of surveillance and antibiotic consumption data 5,6 .

Antimicrobial resistance (AMR) is becoming an increasing burden on healthcare organisations globally 7 , and in Africa and Asia, it is spreading rapidly, due to strains on the healthcare system, and system-related structural and process challenges ${ }^{8,9}$. Critically, it is now being recognized that for actionable and impactdriven AMR research, it is essential to include all stakeholders, including different healthcare professional groups, patients and the public ${ }^{10,11}$. Efforts to address AMR in human populations through interventions that target the prevention (e.g. vaccination, provision of clean water and sanitation, and awareness campaigns), diagnosis (using point-of-care tests and microbiology laboratories to identify causative pathogens), and treatment (prescribing antibiotics) of infections are collectively referred to as antimicrobial stewardship (AMS) ${ }^{12}$.

Antibiotic prescribing is a social act, subject to social norms and the influence of culture and context. Distinct variations in the social norms, values, and behaviours between the surgical and medical teams have been reported, with different approaches to antibiotic decision-making ${ }^{13}$. The surgical teams perceive themselves to be highly skilled interventionists, and attribute value to different outcomes and risks in relation to patient care, for example attributing greater priority to infection prevention than following AMS principles in order to prevent the emergence and spread of AMR in their patient populations ${ }^{13,14}$. This can lead to variation in the antibiotic prescribing patterns, with surgical patients more likely to be on broad spectrum antibiotics for longer duration of time, in patients with no evidence of infection ${ }^{15}$. Most of this evidence is from high income settings, with less known about the processes, behaviours, and drivers for AMS and IPC in surgical populations in lower-resource settings ${ }^{16}$. In LMICs, a lack of adequate healthcare infrastructure and cost implications means that patients may not always have access to healthcare, including surgery ${ }^{17}$. A better understanding of the dynamics of antibiotic use and IPC in surgical populations is as relevant, if not more, in LMICs as it is in high income settings ${ }^{6}$. We report on the findings of an in-depth qualitative study utilizing non-participant ethnographic observations, face-to-face interviews, and in-depth case-studies which include patient and healthcare provider narratives on infection management and antibiotic prescribing in surgical populations across two academic institutions in South Africa (SA) and India.

\section{Methods}

Setting and participants

This study was conducted across adult gastrointestinal and cardiovascular and thoracic (CVTS) teams in two surgery referral university hospitals with established AMS in SA ${ }^{18,19}$ and India ${ }^{6,20}$. Hospital A in 
Cape Town is a 950-bed government funded teaching hospital which in addition to being a tertiary centre, provides non-tertiary services to the local population. Hospital B in Kerala is a not-for-profit charitable 1350-bed tertiary centre.

\section{Inclusion and exclusion criteria}

All healthcare professionals in the selected surgical teams and their patients were eligible to participate in the study.

\section{Sampling, Recruitment and Data Collection}

Between July 2018 and August 2019, researchers conducted an ethnographic study involving nonparticipant observations, interviews, and documentary analyses using a pre-piloted data collection guide 6,13. In India the data were gathered by EC, VN, and S Surendran. In South Africa the data were gathered by $\mathrm{CB}, \mathrm{EC}$ and OM. The surgical leads were invited through the site study leads (MM and SS) to attend face-to-face meetings with the researchers (CB, EC, OM, S Surendran, VN), to be introduced to the research. After the meeting the surgical team leads were emailed the study information sheet and they cascaded these to their teams. Verbal informed consent was obtained before each episode of observation. Written consent was obtained from the patients and staff prior to the interviews. The interviews took place following the observations to ensure that participants could be questioned about their antibiotic decision-making without affecting their behaviours during the observations. Qualitative data were gathered from the teams until saturation was reached and no new themes emerged.

\section{Ethnographic observations}

The ethnographic study design included non-participant direct observations, interviews and documentary analysis (Appendix 1, supplementary data). Researchers conducted the ward observations and face-toface interviews with key informants. Detailed, descriptive notes of observations were collected. Separate reflective notes were kept detailing the observer's perceptions and interpretations of what was recorded. Handover sheets, multidisciplinary team meeting notes and the policy and guidelines on antibiotic prescribing were collected to provide contextual knowledge of the processes. These different methods supported cross-validation and triangulation of the findings.

\section{Face-to-face interviews}

Healthcare professionals and patients (considered to be well enough to be interviewed) participating in the observations were invited to participate in a follow up face-to-face interview. Staff and patients were interviewed at a time convenient for them. The interviews were semi-structured with an interview guide ${ }^{21}$, and were audio-recorded and transcribed verbatim and anonymised prior to analysis. The interviews of patients took place at their bedsides in the hospital or in outpatient clinics, when patients came back for follow up appointments. The interviews were semi-structured with an interview guide for healthcare professionals (Appendix 2, supplementary data), and one for patients (Appendix 3, supplementary data) developed through review of literature, and drawing upon previous work of the research team ${ }^{13}$. 
In India, several interviews were conducted in the Malayalam dialect. These interviews were transcribed in Malayalam, the transcriptions translated to English, and then back-translated again to Malayalam (by VN and S Surendran) for accuracy. One patient interview in SA was conducted in English and Afrikaans and the transcription was translated by CB. All other interviews were conducted in English.

\section{Case studies}

Case studies were generated by in-depth documentary analysis of patient medical records using a piloted template. Data on all variables (e.g., microbiology results, antibiotics prescribed, medical history, surgical interventions) were collected. The healthcare professional, carer and patient experience was captured through focussed interviews.

Analysis

The data were analysed using classic grounded theory approach ${ }^{22}$, using mainly inductive methods of inquiry. Grounded theory relies on simultaneous data collection and analysis, in an iterative manner that enables theory construction and does not rely on existing frameworks for analysis. The analysis aimed to explore categories and relationships within the data collected. During focused coding, a constant comparative method was used for the analysis of the emerging categories and themes ${ }^{23}$ aided by Nvivo 11 software. The data from observations, documentary analysis, and interviews were open coded to identify key categories, which were developed into themes. The analysis was conducted using an iterative and recursive process of moving between the coded data, data collection in the field, and the higher-level themes, until the themes and the relationship between the themes reached saturation (i.e. no new themes or inter-relationships between them were identified).

To mitigate professional biases, our research team included two pharmacists, one anthropologist, one nurse, three infectious diseases specialists, five physicians from a range of surgical specialties (gastrointestinal, general, cardiothoracic and emergency). The diversity of backgrounds enabled us to consider our role-related biases and examine different perspectives throughout the analysis.

\section{Results}

In hospital A (SA), 60 hours of observations and 68 interviews (61 healthcare professionals, 7 patients) were completed. In hospital B (India), over 138 hours of observations and 50 interviews (44 healthcare professionals, 6 patients) (Fig. 1, full anonymised participant details available as supplementary material) were completed.

Six in-depth (two in hospital A, four in hospital B) case-studies were completed. Two illustrative casestudies, one from each hospital are presented in Fig. 2. These case-studies provide a contextual anchor for understanding the findings from the ethnographic study and represent two female patients, who needed surgical intervention and subsequently remained in hospital due to infection related complications and surgical site infection. The purpose of the case studies is to illustrate the complexity in 
infection related care in the surgical pathway, particularly highlighting the number of healthcare professionals involved in the infection and antibiotic decision-making for a patient. Additionally, what the demonstrate is that infections and antibiotic use remain a risk throghout the patient pathway and are not limited to the surgery itself.

The following key themes emerged from the analysis of the data.

\section{Process driven and overlapping/shared roles and responsibilities for inpatient care}

IPC and antibiotic decision-making along the surgical pathway require input, responsibility and action from several key healthcare professionals. Figure 3 illustrates these implicit existing roles of healthcare professionals within the surgical pathway in relation to AMR, AMS and IPC. In practice, these roles and responsibilities overlap across different professions and specialties, and what can occur is a lack of clarity about what needs to be done by whom (Table 1, Quotes 1-3). Apart from removal of lines, the responsibilites in the surgical teams was similar across the two hospitals. The intensive care doctors are well integrated with the surgical teams. Hospital A has a more developed AMS team. 
Table 1

The key emerging themes from interviews and observations, normal text denotes observation notes, italics denotes quotes from participants

\section{Theme Selected Quotes}

Process driven and overlapping/shared roles and responsibilities for inpatient care

\section{Quote 1}

Registrar asks nurse (doing an extra shift from a different ward) whether the social worker had seen the patient as discussed in a grand round. The nurse leaves the bedside to check with ward staff who worked on the previous day. None of them had seen the social worker. A senior nurse (who just returned from leave today) enters the space. She is asked by the registrar, who wants to know who is in charge, why this order was not followed through. The senior nurse explains that she has been away and that the manager is off sick. The registrar mentions a list of people who he will report this to and compares it to another case, where a patient's hospital stay was extended for weeks because a similar issue was not sorted. He says firmly: 'I want this sorted out today!'. Frustrated, he pages through the patient's notes and when he sees the medication charts, he aks: 'Why is this in such a mess? It's like dog meat!'... He then asks if the patient has any lines in situ. The nurse lifts the sheet off the patient's arms and hands, saying she doesn't think so. The researcher points towards an intravenous bag connected to a line running under the sheets down the middle half of the bed. The team follow the line and find that the patient has a central line in the left groin. The registrar asks for the line to be removed.

\section{Observation notes, Hospital A Ward round, CVTS}

\section{Quote 2}

A surgeon's advantage is that, we have a lot of medical background, obviously because we all go through the same training, but unfortunately what the medical doctors lack is the surgical background, which is not easy, I mean if we read up, we can get a lot of medical knowledge, but unless they are in the war zone they won't learn any of the guerrilla warfare. I mean you know it is very difficult for them to actually think in our perspective of things. I might not realize for example, two days back, I operated on a lady for biopsy, infected biopsy and she is off antibiotic now. Move on to day 2 and they started her on cefaperazone-sulbactam, I stopped it yesterday, because the source control is attained, everything is fine, she is not having fever, no tachycardia and no leukocytosis, nothing, CRP is normal, already controlled, we can reserve it for her future.'

\section{Consultant Surgeon (29), Hospital B, GI}

\section{Quote 3}

'You can't do everything yourself. There are other fires to fight and you have to choose which one is going to burn down the forest first and sometimes they (registrars) have done all they can at that time. They've prescribed the antibiotics. Because they've got another patient to see, another patient to book for theatre, another patient needs to be treated before they can go to ICU, so they can't physically follow antibiotics around all the time...so things get weighted. As I say, the patient is ... if sepsis is their main problem and you've addressed it surgically, then the antibiotics need to happen. We think surgery is going to make them better, not the antibiotic, but they work together... we prescribe antibiotics and then we expect it to be done, because I don't administer antibiotics. I don't mix any antibiotics. I've never been shown how 
to do it. I have a reasonable expectation that that is their [nursing] job and that they will see it through.' Consultant Surgeon (26), Hospital A, GI

\section{Quote 4}

Surgeon A suggested to stop antibiotics as procalcitonin is low and cultures are negative. Surgeon B asked the team why an antibiotic was started.

Surgeon A and C said: "patient's platelet was low, and temperature was $99^{\circ} \mathrm{F}$ ". They then had a discussion. Surgeon B wanted to continue the antibiotics for 7 days and Surgeon $C$ also suggested the antibiotics be continued until the central line is removed. Surgeon A however wanted to stop the antibiotics as there was no indication to continue them. Finally, after an intense discussion, Surgeon B convinced Surgeon C that antibiotic should be continued. Surgeon A did not seem convinced by the decision.

\section{Field notes, Hospital B, A three-consultant led ward round, CVTS}

\section{Quote 5}

'One thing it [shared decision making between surgeons] prevents is loose cannons. If I write an antibiotic, somebody else is absolutely free the next day to question, "why is the patient on antibiotics?" And usually if it comes down to an objective analysis, if it is not based on evidence, it tends to be stopped sooner rather than later. For appropriate antibiotic therapy, having a team care model seems to be better than an individual. Individual persons tend to have their biases and when there are no crosschecks, the biases tend to carry on.' Consultant Surgeon (21), Hospital B, GI

Ownership for antibiotic management is focused within specialties

\section{Quote 6}

The consultant and registrar discuss the diabetic therapy for the patient on whom the registrar had performed emergency appendicectomy the previous evening. The registrar) retrieves, from her smartphone, a picture taken during the procedure. She shows the picture to the consultant and other registrars. When the consultant sees the picture, she immediately agrees to start antibiotics, telling the patient that the 'uterus looks infected' and that they were going to give antibiotics and then take out the drip and hopefully she could be discharged the next day. The consultant and two registrars present discuss the antibiotic regimen and the registrar who had operated, prescribes them onto the paper chart. He discontinues the co-amoxiclav and writes up azithromycin, ceftriaxone and metronidazole. He gives the chart to the nurse. She reads it and says that it doesn't make sense as 'they want the drip to come out'. The azithromycin frequency is a stat dose, while no frequency is written for the other two antibiotics. Field Notes, Hospital A, GI ward round

\section{Quote 7}

'I promise you if I operate on a patient or my colleague operated on a patient, the patient must get better. We didn't come here to make the patient sicker. You must have that in your mind. I light the lamp every morning, I pray for the health of my patients because they must go home, they have got families, they have got kids, I mean, what else are we going to do? Did we get into this job just to look at bloods? No, we got in this job to sort something out. That's what's a surgeon's prerogative is, you are here to do something. A surgeon generally is one or two things, either I can help you or I can't help you. If I can help you, I must help you. If I can't help you, I tell you I can't help you. I'll send you for palliation, that's it. We are not physicians; we don't have much of grey, 
we are black and white. I can, I did, I put in a lot of hard effort here, get better, have a good life.'Senior registrar (16), Hospital A, CVTS

\section{Quote 8}

'Surgeons are still very powerful and when the patient is not doing well, sometimes science defies them, and you cannot do anything to stop. You know that what they are saying is not correct, but they are worried because their patients are doing badly, so you start meropenem today, ceftriaxone tomorrow, move on to ertapenem the day after and you are helpless, because the patient is doing badly and nobody can do anything. I think surgeons will have the final call because after all, it is the patient who comes to them and they operate and they are largely responsible for the outcome, however, suggestions [from anaesthetists, AMS, and ICU doctors] and seeing the consistency and inputs, which are there, they probably will build a sense of trust and then inputs could be shared as amicably as possible.' Anaesthetist Consultant (23), Hospital B, GI

\section{Quote 9}

'What we follow is that whoever has operated on that patient, you know he should not feel that somebody else has caused a problem for him, so we do tend to leave it like that, we do not pull rank there like I would suggest that can we stop it, but if he insists, I would not continue at all [to suggest changing antibiotic or stopping it]. Consultant Surgeon (2), Hospital B, GI

\section{Quote 10}

'One of my old seniors used to say, 'antibiotics might make a third-class surgeon a second-class surgeon, but they never make a second-class surgeon a first-class surgeon'. I know it is that fear that let me not to take a chance. I do not think it is a challenge rather, you know, fear that their own surgery will be inadequate. Most of them tend to be quite confident about the surgery, but they, we always have this feeling that something else, is going to make a difference in our results. Overtime when they see that it has not made a difference or they have seen across the spectrum, they tend to change those habits. I think it is more that fear of 'let me not to take a chance and something might go wrong' which is there.' Consultant Surgeon (2), Hospital B, GI

\section{Quote 11}

'The registrars (junior doctors) would be the ones, you are doing a ward round you see a wound that is not looking so good and you start antibiotics. You do the cultures and everything and you start antibiotics. The consultant will come in and say that perhaps these antibiotics are not a right choice, we should change it to this or discuss it with microbiology.' Surgeon, Hospital A, GI

\section{Quote 12}

And then if this [antibiotic prescription] happens in ICU a decision will be taken by the intensivist. They are the ones who will initiate antibiotics in most cases. And then if a decision is taken on the ward round, it is usually the intensivist together with the consultant cardio thoracic consultant who is there in that ward round. Surgeon (14), Hospital A, CVTS

\section{Quote 13}




\section{Theme}

Hierarchies restrict

the integration of

Infection

prevention and

control practices

\section{Selected Quotes}

'We are working together as a team; that is a check. If I get overwhelmed by a patient's situation and write something which is not rational at that point of time, for example, if I write a dose of colistin or polymyxin or tigecycline for a patient who probably does not need it, somebody in my team will sound it in the next day, so one dose would go but by then some discussions definitely arise, usually in ICU. So multiple discussions like that has now resulted in a situation where.... there is a restraint, especially in my team I have seen a restraint because there will be a fight otherwise, but many a time, these things are overlooked.' Consultant Surgeon (11), Hospital B, GI

\section{Quote 14}

'If the registrar initiated it we would continue it but we would also consult microbiology... that's why we have rounds with microbiology so that we can consult and streamline because there is no use going broad-you need to be focused and that's the big thing about our antibiotic protocol.' Senior Registrar (16), Hospital A, CVTS

\section{Quote 15}

One of the ICU consultants and one of the Gl-acute surgeons discuss the patient's progress and plan. The surgeon puts on an apron, ties it at the back and then puts on gloves and goes around to the patient's bedside. He reaches out with his long-sleeve-clad arm and gloved hands to assess the patient's wound, moving some pipes aside. The GI intern goes to the patient's bedside, to help the surgeon. The ICU consultants slaps his hand away playfully, and tells him, 'You don't touch a patient in the ICU without gloves and aprons, especially this patient with Acinetobacter and Klebsiella. The intern remarked that this ICU works differently from where he normally works. The ICU consultant responds, saying that this is how he should work. The intern laughs and tells consultant that she must tell them. The consultant returns, 'I'm telling you, take it back to them'. Observation notes, Hospital A, GI ICU ward round

\section{Quote 16}

'We're generally fairly lackadaisical with hand washing, and barrier protection, so people will go into the room [patient isolation rooms] and it is a problem. I think I find the issue of, putting on aprons and gloves and not sitting on the patient's bed is, you know you lose something. You know if you sit on the patient's bed without aprons and gloves you have a much closer contact with the patient and it's, you know it's getting in the way of that relationship. And I think that, perhaps, when we have a colistin resistant drug, or a pan resistant bacterium, if there were people walking around in space suits everyone would take it a lot more seriously. But we've got a kind of like a blue apron and pair of gloves, perhaps the kind of whole HIV epidemic and our dealing of that is that we've become quite casual with infectious diseases. I think we are more casual than we should be. We've got a major problem with nursing ... they're overrun. There is just not enough of them... and the leadership from nursing isn't.. to me, you need a head-sister not letting doctors into the room unless they've got their apron on, and that sort of, it doesn't happen too much. I don't think they'd [doctors to head nurse] respond to it positively at all but they would do it.' Consultant Surgeon (11), Hospital A, GI

\section{Quote 17}

'We are auditing who are all the people touching the patients without hand washing. My name has come up many times, and all my colleagues' names are there and a lot of anaesthesia colleagues also. One thing is that they know 
the nurses should be more proactive, and they should have a little more decision-making ability. They should say that okay, no you are a doctor, you have not washed your hands, so please wash your hand before touching. They are not able to say that. Why they are not able to say that is probably the hierarchy and their lack of confidence. See, if you are a nurse with a 10 to 15 years' experience in the ICU, then you will have the confidence to say. If you are a nurse who has just came out from the nursing college and joined the cardiac surgery ICU, then they are scared to talk. There is basically a lot of fear.' Consultant Surgeon (15), Hospital B, CVTS

\section{Quote 18}

'The main source of infection is from the caregiver when they downplay the importance of handwashing and sometimes when the physician or surgeon is asked to have a hand wash before touching the patient, it may not end on a sweet note, but it plays a major role. If my primary worry is regarding the patient, definitely we will follow this and, in our ICU, each staff is assigned a particular patient for a few set hours. If some clinician is coming in and meeting that patient and if they are not washing their hand immediately, the nurse is noting down the person's name and usually by the end of the week, this information is passed from the head of the department down to every one saying you are scrutinized. So, you do not want to hear your name repeatedly for the same mistake.' Consultant Surgeon (14), Hospital B, I14 CVTS

\section{Quote 19}

'Usually in surgery, main thing is that [to make sure] we are changing the dressings well, we are removing the drains. Those are the most important when sisters are giving drugs [through] IV [intravenous] lines, when they are taking care of the central lines and IV lines. Those are the most important things. Sisters are the ones who are going to give the $I V$, they are using the central line. They are going to give all the drugs, so they should be more conscious. They should wash their hands before touching the patient before giving the IV drugs. After giving drugs also they should wash because there is high chance that they can [introduce] infection directly to the central line which can cause blood borne sepsis, so that is more important, central line care and IV-line care. Then after that the wound infections and wound dressings while doing the dressings. Those two are the important ones.' Registrar (7), Hospital B, GI

\section{Quote 20}

We tell them [to practice IPC precautions] but they [doctors] just ignore us sometimes. Sometimes you meet difficult doctors and you see you can't tell him or her. Then you just leave it. It is difficult. Nurse (34), Hospital A, GI

\section{Quote 21}

"If it is in a ward, we can say the nurse's role is important [ in infection control practice], but here in ICU, we can't say that only nurses role is important. It is true that nurses are the one who administer the medications and other things, central line use is more for nurses etc. but when there is an emergency, sometimes doctors do administer the medicine or doing an echo, like this many people are involved. So I feel, in the ICU both doctors and nurses have an equal role in the infection control practices." staff nurse (34), Hospital B, CVTS

\section{Quote 22}


Theme

\section{Selected Quotes}

When the nurse has been assigned a patient, the nurse can always tell anybody; it may be a doctor or any technician or anybody, if they are not following the infection control practice before touch his/her assigned patient, they can directly tell them to follow... If the staff is less experienced, maybe below 6 months, they may not tell. They would be scared or hesitant to tell, but we train them to tell directly. Staff nurse (37), Hospital B, CVTS 


\section{Theme}

Patients as drivers for antibiotic prescribing decisions

\section{Selected Quotes}

\section{Quote 23}

And you are all talking about evidence-based medicine, but in India what matters is a socioeconomic factor. Socioeconomic factors decide the choice of therapy in most cases, not evidence based medicine and who is going to make that socioeconomic, patients won't make, you have to as a doctor, you have to make that choice whether this is good for our patient, you know, or whether I should cut down this and give this.

\section{Consultant Surgeon (15), Hospital B, CVTS}

\section{Quote 24}

If we had started IV antibiotic from the beginning, probably she would have improved quicker. We had initially begun treatment with IV antibiotic but since she could not afford it, it was changed to oral. Even though the microbiologist had suggested [stronger] antibiotics, we could not prescribe those as she wasn't willing to buy them. We prescribed a [stronger] antibiotic towards the end when we could arrange it for free from the hospital pharmacy. However, this could only be given for 2 days.' Surgeon (TBC), Hospital B, GI

\section{Quote 25}

'The finance aspect plays a big role for selecting the antibiotic; ... so in some patients who have complete financial restraints, we will have to maybe reduce the doses or take into consideration other drugs, which can cover the bacteria even though if it is not sensitive. So in those cases, the other thing that happens is they will want to go to another hospital, probably somewhere in medical college where the medicine is free.'Pharmacist, Hospital $\mathbf{B}, \mathbf{G I}$

\section{Quote 26}

'The surgeon informed me that I have a hernia. He said that a surgery would be required but it is not urgent [...] Surgeon said that with medicines, the surgery could be postponed up to 3 months later. Since the surgery is inevitable, I wanted it to be done immediately as it would be difficult for me to get leave later from the firm where I am working.'Patient 47, Hospital B.

\section{Quote 27}

'You need to talk to [patients] I think, you need to tell them that: "okay you have a fever but the fever need not necessarily mean the presence of an infection... however, if you develop certain signs of an infection, then do come back to me and in that case I would definitely start an antibiotic for you"... basically to convince the patient. If I am able to convince the patient, the patient will go back happily, if I am not able to convince the patient, the patient will go from me to another surgeon, and to another surgeon, ultimately to a surgeon who will actually prescribe an antibiotic and then he would be happy, so he will go off doctor shopping.'Surgeon, (6) Hospital B, GI

\section{Quote 28}

'Patients don't give a history of disease, but a history of antibiotics.' Surgeon (6), Hospital B, GI 
The surgical teams are prone to respect the decisions of their peers (Table 1, Quotes 4-5). Identifying these implicit existing roles is important, as historically most AMS interventions target junior doctors, bypassing the existing roles that a wide range of healthcare professionals identify as theirs in relation to IPC and AMS (Table 1, Quotes 2-3), including the influence of the senior surgeons on one another.

\section{Ownership for antibiotic management is focused within specialties}

The bulk of decision-making about IPC and antibiotic use remains with the surgical and intensive care unit (ICU) teams; the pharmacists have a limited role. Nurses are not involved in decision-making, though they are responsible for the administration of antibiotics. An important factor highligted by nurses in hospital A is how direct communication, or the lack thereof, between the prescriber and nurses could impact on how quickly a new antibiotic was administered (Table 1, Quote 6).

The anaesthetists are recognised as being responsible for ensuring timely surgical prophylaxis in the operating room. Post-operative care is administered in the ICU or surgical wards, depending on the type of surgery and patients' condition (Fig. 2). Though the intensivists take major decisions whilst in ICU, the ownership for the post-operative decisions related to antibiotics can be tenuous at times, even within surgical teams. These decisions reflect firmly held beliefs that the surgeon who had operated on the patient should retain ownership of decision-making as they are ultimately responsible for patient outcomes. In India, other surgical colleagues, would avoid challenging or intervening in the patient's care unless invited to do so by the operating surgeon, while in SA decisions on antibiotic therapy are made by the intensivist but in collaboration and discussion with the operating surgeon (Table 1, Quote 7-9).

The uncertainty and fear of failure does to some extent influence how the surgical team deliberate their decisions on antibiotic management, viewing antibiotics as a safety net more than a cure for infection (Table 1, Quote 10). The choice of post-operative antibiotics and targeting of therapy is predominantly driven and monitored by senior surgeons (Table 1, Quote 11). A similar approach is followed in the decision to send for culture and sensitivity. These decisions are often initiated by the junior surgeons but a quick account is sought from and/or by the senior team. Follow up of cultures and communication with microbiology is most often enacted by the trainee surgeons. However, decisions related to the removal of indwelling catheters and lines are initiated by senior surgeons. Whilst the ledership for surgical decisionmaking is clear, there are gaps in the leadership for IPC and AMS, outside of the ICU setting within surgery.

The most multidisciplinary and integrated model of care observed was in the ICU setting in both hospitals, where the surgical, intensivist, nursing, and pharmacy (in GI India) teams together with microbiology and infectious diseases services come together to deliberate about the infection-related care of patients. This shared responsibility between the ICU and surgical teams ensures patients are promptly diagnosed with infections and treated appropriately. The team dynamic between the specialties does ensure that antibiotic decisions for patients in the ICU get thoroughly reviewed and questioned and 
surgeons recognise this can have a positive impact on their antibiotic management (Table 1, Quotes 1213).

The infectious diseases and microbiology teams provide advice on infection management and antibiotic use in the surgical pathway and the surgical teams do recognise the input from microbiology to assist in targeting antibiotic therapy through the multi-disciplinary ward rounds. Their input however is viewed as a consulting service and the antibiotic decision-making for surgical patients (in the surgical ward) remains within the surgical teams (Table 1, Quote 14). This can also affect the leadership for IPC and AMS decisions.

\section{Hierarchies restrict the integration of infection prevention and control practices}

Despite available policies and guidelines on IPC practices across both sites, the patient case-studies (Fig. 2 ) highlight the vulnerability of surgical patients to post-operative infections, and the complexity of care as multiple healthcare professionals become involved in their care as they move through the pathway. Organisational policies and expectations in both settings considers IPC the responsibility of all staff. In practice, however, there is significant variation in terms of how much staff engaged with IPC as a core part of their role, or considered it their responsibility (Table 1, Quotes 15-19). As in the previous theme, the most integrated care around IPC is in the ICU, where teamwork is more cohesive and nursing staff are more empowered to participate in and amplify their concerns for IPC processes (Table 1, Quote 16). Adherence to hand hygiene was sporadic however across all specialties and units, and often not performed at the right moments in patient care (Table 1, Quote 14). For example, on many occasions on ward rounds, the senior surgeons would use alcohol gel between patient consults, though they had not touched the patient for examination, performed a procedure or touched the patients' surroundings whilst the junior doctors who had been carrying out tasks and examinations would sometimes not adhere to hand hygiene. The responsibility for promoting and monitoring IPC is apportioned to nursing staff, who often due to entrenched hiearchies may not feel able to challenge deviations from expected behaviour (Table 1, Quotes 18-22). The existing hierarchies mean that often (outside of ICU setting espcially), it is the head nurse or sister who is relied upon to feedback on IPC behaviours to the surgical teams, causing a delay in correcting behaviours and ensuring that for each patient the correct processes are followed (Table 1, Quote 16-18). The use of contact precautions and being placed in isolation rooms can have consequences for patients who may feel that they are responsible for their infection as attested to by the patient in hospital A (Fig. 2, case study A). Additionally clinicians see it as a barrier to patient rapport (Table 1, Quote 16).

\section{Patients as drivers for antibiotic prescribing decisions}

The patient is the one constant along the surgical pathway; however, the patient role in AMS is not well understood. Healthcare staff recognise that they need to consider individual patient factors, other than purely clinical ones, and that the patient can influence their decision-making. In hospital B, clinicians recognise the need to consider the financial implications of treatment decisions for patients, as patients 
are paying out of pocket or need procedures not covered by their insurance. In this case, therapy is often tailored to meet their financial capability (Table 1, Quotes 23-24). Treatment decisions also influence care, i.e., if treatment is expensive, this may result in patients having to be transferred to state hospitals once their personal funding is depleted (Table 1, Quote 25). Where patients are able to choose the healthcare provider, it is difficult for clinicians to negotiate with patients to delay antibiotic treatment or take a more conservative approach to antibiotic use, as patients could choose to go elsewhere for their care if they were not satisfied (Table 1, Quotes 25-28).

\section{Discussion}

IPC and AMS remain key priorities for safe surgery. Central to this is the need for integrated pathways, where the IPC and AMS related roles and responsibilities of all healthcare professionals are harnessed and amplified so that they can act according to the expectations placed upon them. The day to day care in surgical pathways is the responsibility of the surgical teams and as important as designated IPC and AMS teams are, there needs to be greater recognition of the role of some staff (particularly nursing staff) within surgical teams. Nurse leadership and other stakeholder engagement need to be developed to enable full integration in care pathways. Many lessons can be learnt from the integrated models of care in the ICU setting.

Focusing on the key actors involved in IPC and antibiotic decision-making, in surgical pathways in institutions leading the AMS effort in their respective countries we propose a framework for integrating AMS and IPC in surgical teams (Fig. 4). Adopting a framework approach will help identify and fully utilise the existing roles of surgical team members within AMR, AMS and IPC programmes. Engendering a sense of responsibility to ensure consistent practices in relation to IPC and AMS, requires clarity on expectations from staff and defining explicit roles and responsibilities. This can be supported by a better understanding of where individual staff feel responsibilities lie, and how they see their role in the process. Nurses have an implicit role to play in effective antibiotic management and IPC, though this role is often not recognized by the wider team. An integrated approach to IPC and AMS across surgical pathways to ensure effective and safe care can only be developed when we build leadership from across disciplines, including nursing and pharmacy. Whilst stewardship teams can initiate this process, successful outcomes can only be achieved when there is local ownership for IPC and AMS within surgical teams. To support this we propose to broaden the definition of surgical teams to include pharmacy and nursing staff.

In both hospital sites, there exists AMS targeting the surgical teams. These programmes, however, tend to target junior doctors, overlooking the collective nature of stewardship and the important role played by senior surgeons in overseeing the pathway and making critical decisions. This finding and adds to the existing evidence ${ }^{13,14}$. This may speak to a universal truth in surgery in that there is generally a named surgeon who is responsible for the outcome of a patient (and many accept that that is important) and yet individual surgeons cannot be at the bedside 24/7 (and neither can members of her/his team) - and 
therefore we need a shared responsibility for many decisions. Not just a shared responsibility within the surgical teams but also better team working and sharing of responsibility across professionals.

The similarities in these surgical pathways across two different specialties and two different countries highlights how power of social norms, culture and hierarchies can determine IPC and antibiotic prescribing behaviours. In the previous study conducted by the authors investigating antibiotic stewardship interventions from the perspective of AMS teams in different resource settings, we reported a lack of engagement with surgical teams in $\mathrm{AMS}^{6}$. High income settings had more individual parameters within their existing AMS programmes, whereas low income settings were only able to implement a limited set of interventions chosen opportunistically and in accordance with existing means. For effective and sustainable AMS, it is critical that interventions consistently identify the actions and behaviours that need to be adopted by each individual in the pathway. When developing AMS programmes for surgical teams, the proposed framework can be applied to ensure that the key components necessary for sustainable change are addressed. This framework builds on research from united kingdom, as well as SA and India. What has been demonstrated in this study is that regardless of the setting the surgical specialty has common themes in relation to antibiotic decision making and IPC. As such this framework could be applied to surgical teams across different settings.

To date, AMS programmes in hospitals have focused on medical specialties and taken a one size fits all approach. This means that often an expectation is placed on healthcare professionals to adopt behaviours which may not be appropriate for the challenges they face within their practice and the resources available to them. Interventions in antibiotic prescribing have considered only one point at a time in the patient pathway, and in the case of surgical patients, attention has been focused primarily on prophylactic antibiotic use. As illustrated by the case studies, post-operative antibiotic prescribing needs to be the target of AMS. The expectation placed on surgical teams to optimize antibiotics needs to be managed in the context of the way that the teams work and the roles and responsibilities that individuals have in relation to infection diagnosis and antibiotic decision-making. Patients and carers' roles in selfcare and their experience of infection related care in the surgical pathway also needs greater recognition. For optimised adherence and IPC, the patient and their carer or family members should be informed about the correct IPC measures and why it is important to adhere to them. The dynamic of patient participation in and expectation of care is further complicated when the patients are paying for the healthcare services they receive. The expectation to be given effective therapies often includes an expectation to be prescribed antibiotics. An understanding of the wider social and cultural determinants is required in order to effectively foster an environment where patients can be more involved in their own care. This is important in the pre-operative preparation and post-operative wound care and recovery process. These findings are particularly pertinent in the current viral severe acute respiratory syndrome coronavirus pandemic which has highlighted the not only the critical need for IPC and AMS measures but has identified surgical populations at increased risk of poorer infection outcomes ${ }^{24}$. Applying a framework approach to integrating IPC and AMS practices in surgical pathways will help address these 
challenges and assure safe surgical outcomes and track the long-term impact of this pandemic on infection related outcomes.

\section{Limitations}

Collecting data across different healthcare organisations, using multiple researchers can pose challenges to systematic comparison of the data. To mitigate against this, we employed a rigorous extended-period face-to-face training for the researchers to ensure consistency in the data gathering and analysis process.

This study was conducted across surgical pathways in two large academic institutions with established stewardship programmes. The findings may not necessarily be generalizable to other hospitals in South Africa or India, where environment and resources, including the availability and level of staff training, and increased demand on healthcare services, compounded by the limited infrastructure, may present additional, significant AMS and IPC challenges.

\section{Conclusions}

Effective and sustainable integration of optimised IPC and AMS practices in surgical pathways needs a framework approach that recognises the structural foundations to support staff to be able to change their behaviours. We need to broaden the definition of surgical teams to recognise the role that nurses and pharmacists can play in surgical patient care, leading to optimised outcomes. We can optimise practices if we are able to implement inter- and intra-disciplinary models of care which recognise the critical need for co-management of surgical patients across professional boundaries and across the surgical pathway.

\section{Abbreviations}

\section{AMS}

Antimicrobial stewardship

\section{CVTS}

Cardiovascular and thoracic surgery

ICU

Intensive care unit

IPC

Infection prevention and control

GI

Gastrointestinal

HREC

Human research and ethics committee

LMICs

Low- and middle-income countries

\section{SA}




\section{Declarations}

\section{Availability of data and materials}

All data gathered or analysed during this study are included in this published article [and its supplementary information files].

\section{Ethics approval and consent to participate}

This study was approved by the University of Cape Town Human Research Ethics Committee (HREC ref. 499/2018) and the Amrita Hospital Institutional Research and Ethics Committee (IEC-AIMS-2018INECONT-005A). The consent to participate information is provided in the methods section.

\section{Consent for publication}

All participants provided consent to participation, and for their anonymised data to be used in the analysis, and in subsequent dissemination for research including peer reviewed publications.

\section{Author contribution statement}

Conceptualization: Alison Holmes, Carolyn Tarrant, Marc Mendelson, Sanjeev Singh, Andy Leather, Mark Hampton, Esmita Charani

Data curation: Sanjeev Singh, Marc Mendelson, Surya Surendran, Candice Bonaconsa, Oluchi Mbamalu, Vrinda Nampoothiri, Adam Boutall, Mark Hampton, Puneet Dhar, Tim Pennel, Esmita Charani.

Formal Analysis: Carolyn Tarrant, Marc Mendelson, Sanjeev Singh, Surya Surendran, Candice Bonaconsa, Oluchi Mbamalu, Vrinda Nampoothiri, Andy Leather, Tim Pennel, Adam Boutal, Esmita Charani.

Methodology: Carolyn Tarrant, Alison Holmes, Esmita Charani.

Validation: Sanjeev Singh, Marc Mendelson, Surya Surendran, Candice Bonaconsa, Oluchi Mbamalu, Vrinda Nampoothiri, Adam Boutall, Mark Hampton, Puneet Dhar, Tim Pennel, Esmita Charani.

Visualization: Surya Surendran, Candice Bonaconsa, Oluchi Mbamalu, Vrinda Nampoothiri, Esmita Charani.

Writing - original draft: Sanjeev Singh, Marc Mendelson, Surya Surendran, Candice Bonaconsa, Oluchi Mbamalu, Vrinda Nampoothiri, Esmita Charani.

Writing - review and editing: Sanjeev Singh, Marc Mendelson, Surya Surendran, Candice Bonaconsa, Oluchi Mbamalu, Vrinda Nampoothiri, Adam Boutall, Mark Hampton, Puneet Dhar, Tim Pennel, Carolyn Tarrant, Alison Holmes, Andy Leather, Esmita Charani. 
This was a cross-disciplinary and international study. All authors have read and give approval for the final version of the manuscript to be submitted.

\section{Competing interests}

All authors have completed the ICMJE form for uniform disclosure Form for Disclosure of Potential Conflicts of Interest and declare. The authors declare that they have no competing interests.

\section{Funding}

This work was supported by Economic and Social Science Research Council (ESRC) and the National Institute for Health Research, UK Department of Health [HPRU-2012-10047] in partnership with Public Health England.

\section{Acknowledgements}

We acknowledge the support and contribution of all the healthcare staff and patients in both study sites in allowing us to conduct this study across their teams. This study is part of the ASPIRES project (Antibiotic use across Surgical Pathways - Investigating, Redesigning and Evaluating Systems) (https://www.imperial.ac.uk/arc/aspires/). ASPIRES aims to address antimicrobial resistance and improve clinical outcomes optimising antibiotic usage along surgical pathways. 'The support of ESRC as part of the Antimicrobial Cross Council initiative supported by the seven UK research councils, and also the support of the Global Challenges Research Fund, is gratefully acknowledged'.

\section{References}

1. Kruk ME, Gage AD, Arsenault C, et al. High-quality health systems in the Sustainable Development Goals era: time for a revolution. Lancet Glob Health. 2018;6(11):e1196-252.

2. Bhangu $A$, Ademuyiwa $A O$, Aguilera $M L$, et al. Surgical site infection after gastrointestinal surgery in high-income, middle-income, and low-income countries: a prospective, international, multicentre cohort study. Lancet Infect Dis. 2018;18(5):516-25.

3. Biccard BM, Madiba TE, Kluyts HL, et al. Perioperative patient outcomes in the African Surgical Outcomes Study: a 7-day prospective observational cohort study. Lancet. 2018;391:1589-98.

4. Allegranzi B, Nejad SB, Combescure $C$, et al. Burden of endemic health-care-associated infection in developing countries: Systematic review and meta-analysis. Lancet. 2011;377:228-41.

5. Schellack N, Benjamin D, Brink A, et al. A situational analysis of current antimicrobial governance, regulation, and utilization in South Africa. Int J Infect Dis. 2017;64:100-6.

6. Charani E, Smith I, Skodvin B, et al. Investigating the cultural and contextual determinants of antimicrobial stewardship programmes across low-, middle- and high-income countries-A qualitative study. PLoS One. 2019;14:e0209847. 
7. Leung E, Weil DE, Raviglione M, et al. The WHO policy package to combat antimicrobial resistance. Bull World Health Organ. 2011;89:390-2.

8. Nejad Bagheri, Allegranzi B, Syed BB, Ellis BPD. Health-care associated infection in Africa: a systematic review. Bull World Heal Organ. 2011;89:757-65.

9. Charani E, Ahmad R, Tarrant C, et al. Opportunities for system level improvement in antibiotic use across the surgical pathway. Int J Infect Dis. 2017;60:29-34.

10. White A, Hughes JM. Critical Importance of a One Health Approach to Antimicrobial Resistance. Ecohealth. 2019;16(3):404-9.

11. Rawson TM, Castro-Sánchez E, Charani E, et al. Involving citizens in priority setting for public health research: Implementation in infection research. Health Expect. 2018;21(1):222-9.

12. Charani E, Holmes A. Antibiotic Stewardship-Twenty Years in the Making. Antibiotics. 2019;8:7.

13. Charani $E$, Ahmad R, Rawson $T$, et al. The differences in antibiotic decision-making between acute surgical and acute medical teams - an ethnographic study of culture and team dynamics. Clin Infect Dis. 2019;69(1):12-20.

14. Charani E, Tarrant C, Moorthy $\mathrm{K}$, et al. Understanding antibiotic decision making in surgery - a qualitative analysis. Clin Microbiol Infect. 2017;23(10):752-60.

15. Charani E, Barra E, De, Rawson TM, et al. Antibiotic prescribing in general medical and surgical specialties: a prospective cohort study. 2019;6:1-10.

16. Meara JG, Greenberg SLM. Global surgery as an equal partner in health: No longer the neglected stepchild. Lancet Glob Heal. 2015;3:1-2.

17. Rayne S, Burger S, Van Straten S, et al. Setting the research and implementation agenda for equitable access to surgical care in South Africa. BMJ Glob Heal. 2017;2(2):e000170.

18. Boyles TH, Whitelaw A, Bamford C, et al. Antibiotic stewardship ward rounds and a dedicated prescription chart reduce antibiotic consumption and pharmacy costs without affecting inpatient mortality or re-admission rates. PLoS One. 2013;8:1-7.

19. Boyles TH, Naicker V, Rawoot N, et al. Sustained reduction in antibiotic consumption in a South African public sector hospital; Four year outcomes from the Groote Schuur Hospital antibiotic stewardship program. South African Med J. 2017;107(2):115-8.

20. Singh S, Menon VP, Mohamed ZU, et al. Implementation and impact of an antimicrobial stewardship program at a tertiary care center in South India. Open Forum Infect Dis. 2018;6(4):ofy290.

21. Charani E, Castro-Sanchez E, Sevdalis N, et al. Understanding the determinants of antimicrobial prescribing within hospitals: The role of "prescribing etiquette. Clin Infect Dis. 2013;57:188-96.

22. Strauss A, Corbin J. Basics of Qualitative Research: Techniques and Procedures for Developing Grounded Theory. London: Sage Publications; 1998.

23. Glaser B, Strauss A. The Discovery of grounded theory: Hawthorne. NY: Aldine Publishing Company; 1967. 
24. Archer JE, Odeh A, Ereidge S, et al. Mortality and pulmonary complications in patients undergoing surgery with perioperative SARS-CoV-2 infection: an international cohort study. Lancet. Epub ahead of print 2020. DOI: 10.1016/S0140-6736(20)31182-X.

\section{Figures}

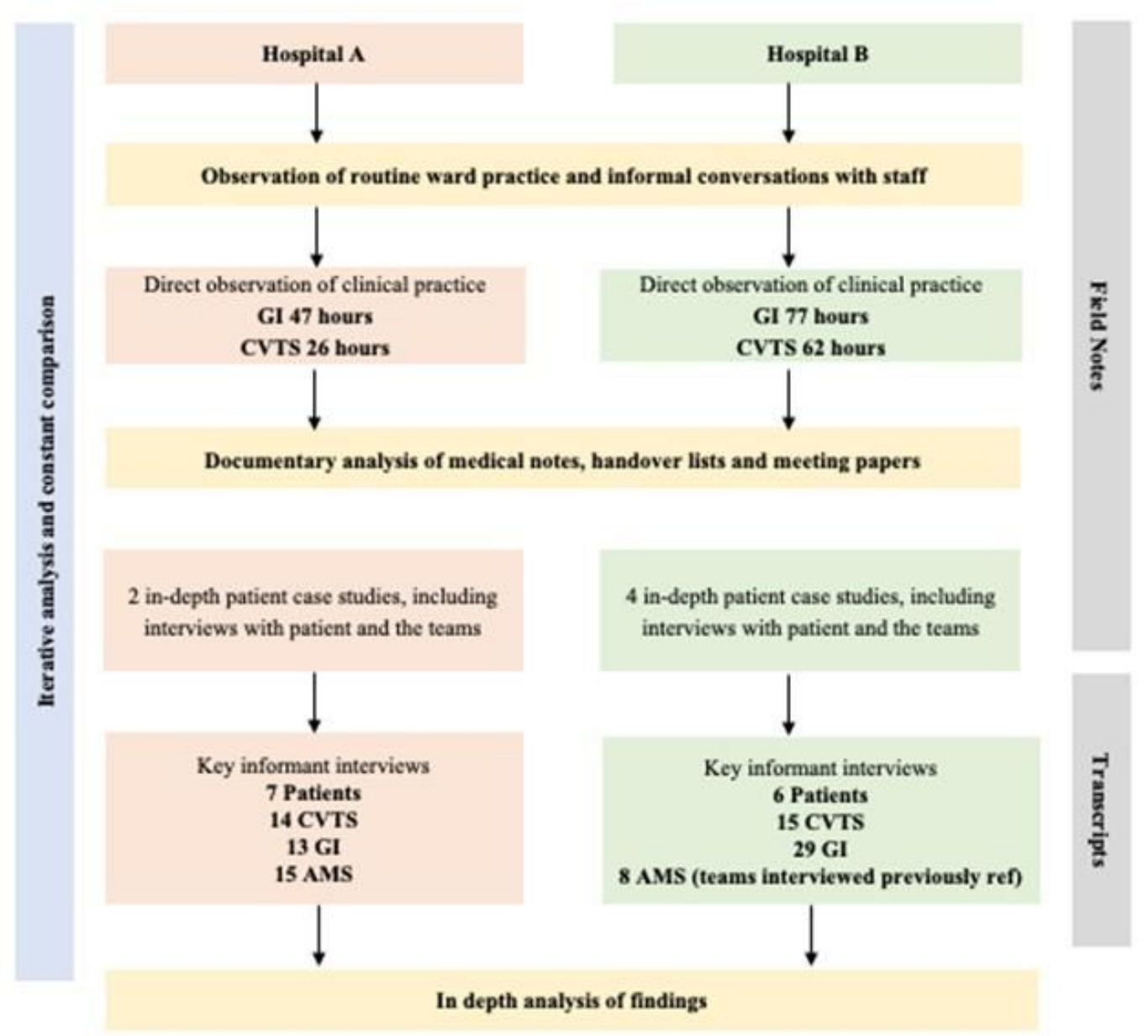

\section{Figure 1}

The data gathering and analysis process 
llospital A

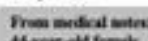

Hyew th thenk.

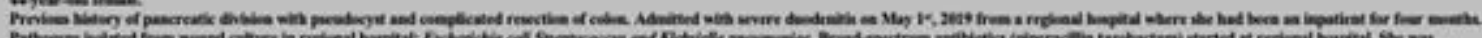
Ph

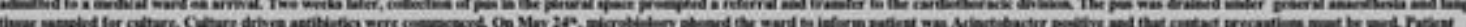

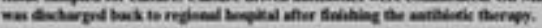

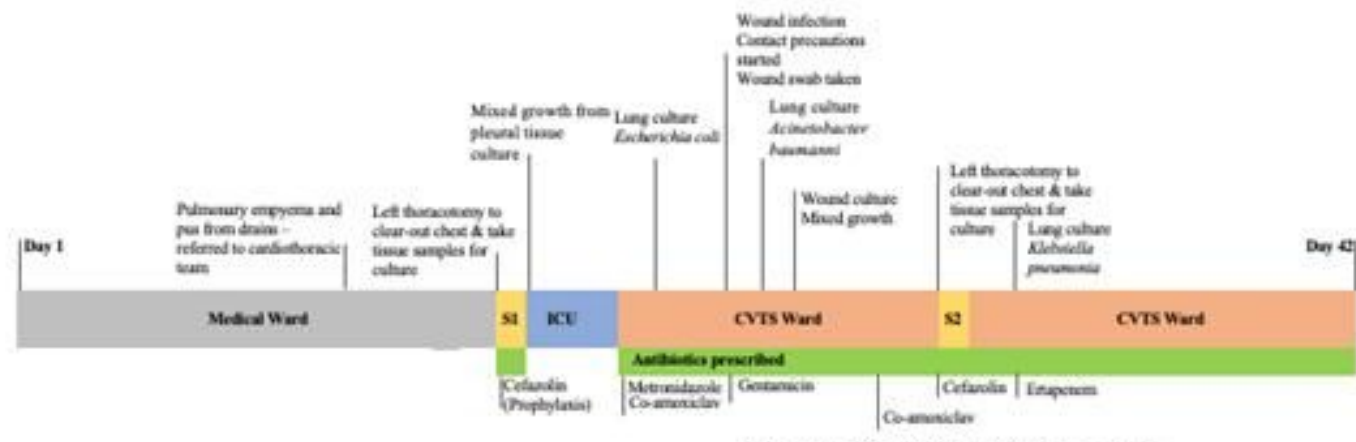

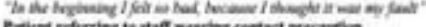

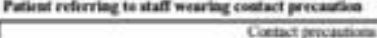

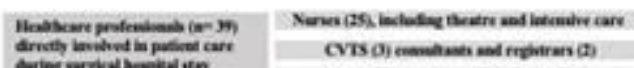

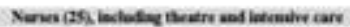

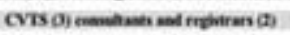

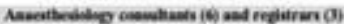

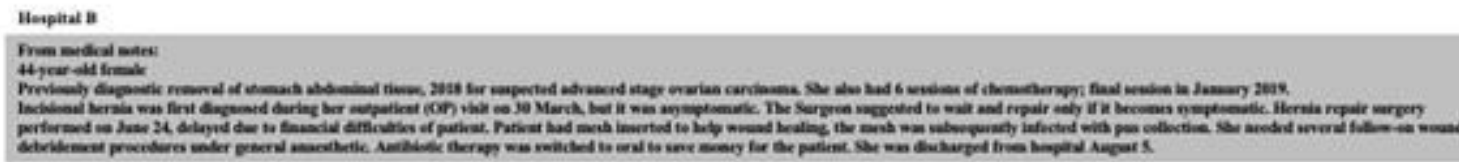

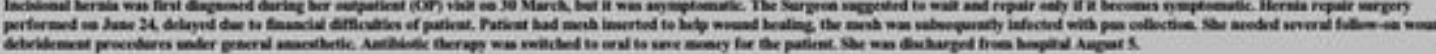

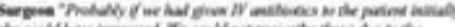

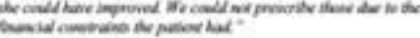

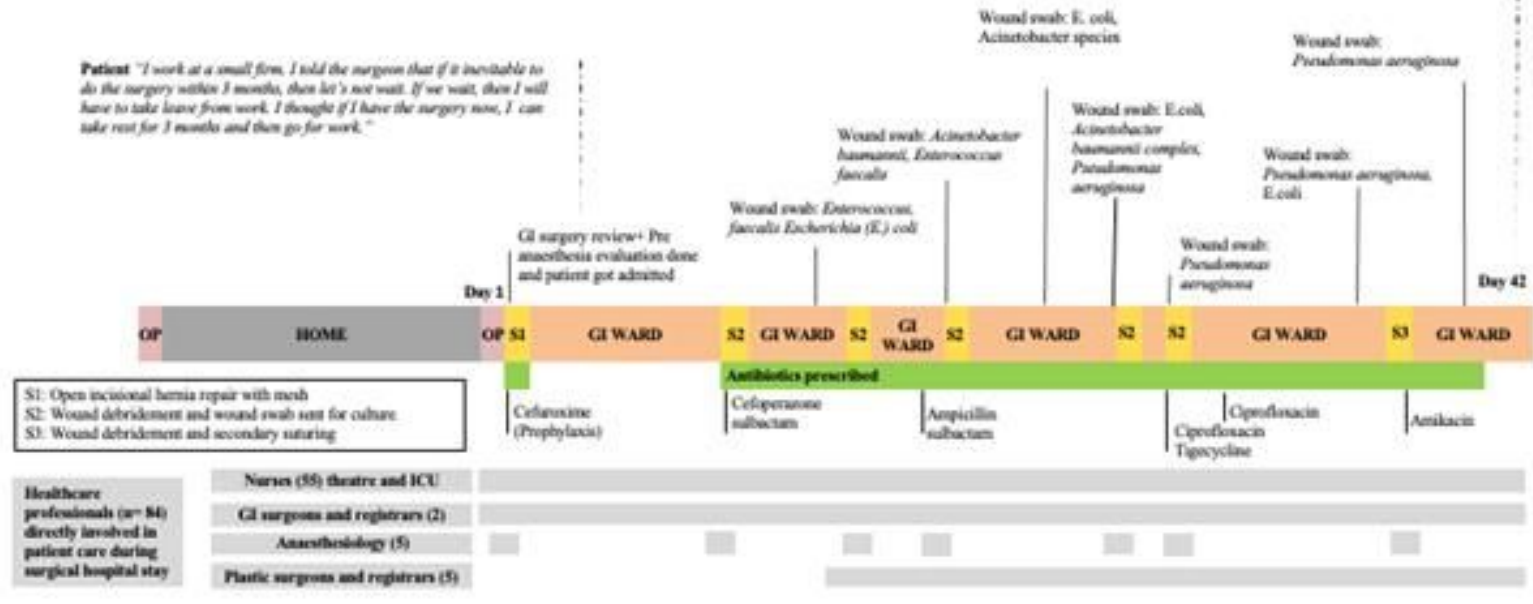

Figure 2

The illustrated surgical pathway for two patients in SA and India 2 a) Case study hospital A 2 b) Case study hospital B 


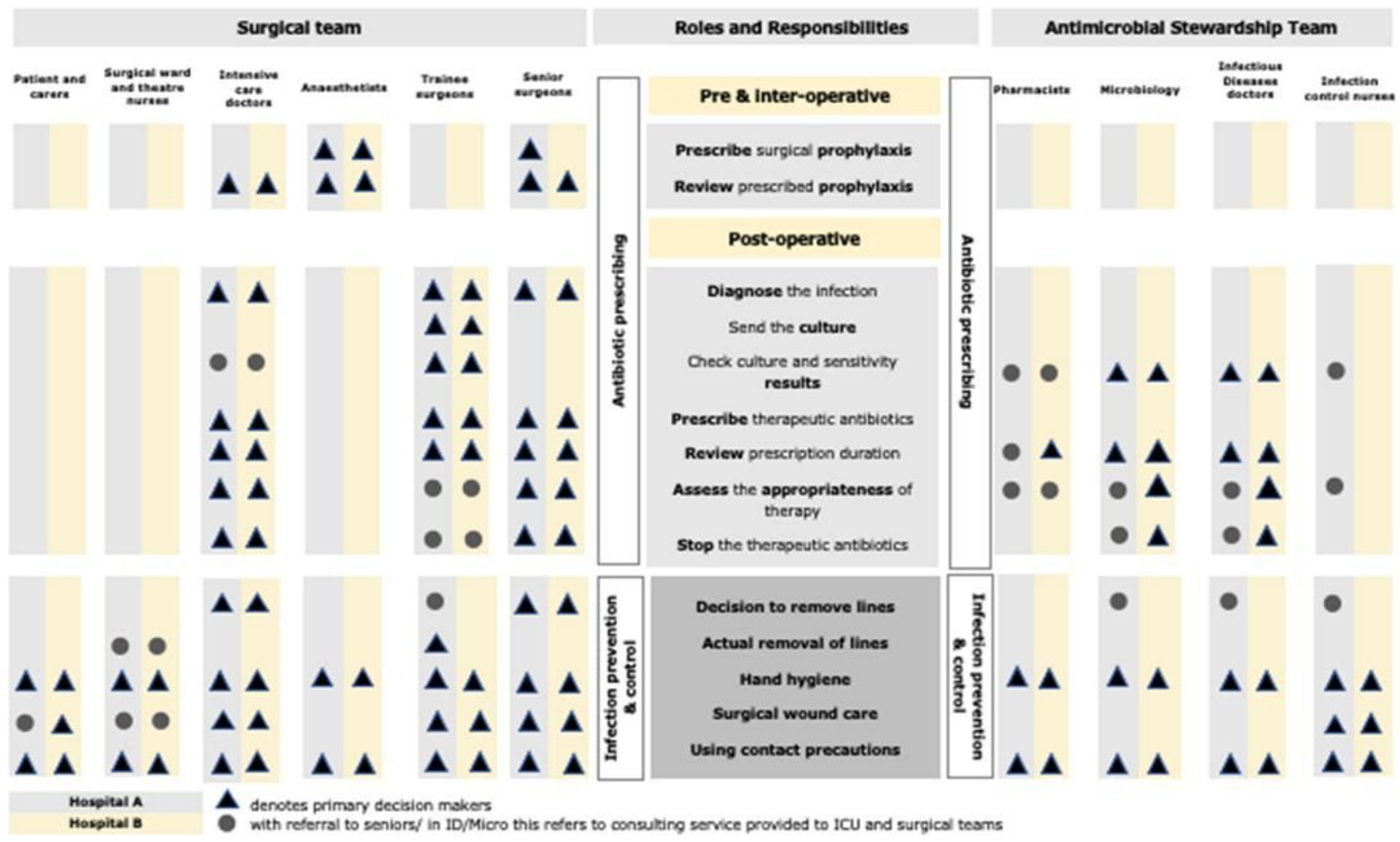

\section{Figure 3}

Roles and Responsibilities in relation to antibiotic prescribing and infection prevention and control

\begin{tabular}{|c|c|}
\hline \multicolumn{2}{|c|}{ STRUCTURAL } \\
\hline $\begin{array}{l}\text { Building Workforce Capacity } \\
\text { Dedicated AMS and IPC team members } \\
\text { working with surgical specialties; } \\
\text { Broaden the definition of surgical teams to } \\
\text { include pharmacists and nurses; } \\
\text { Identify explicit IPC and AMS roles and } \\
\text { responsibilities for surgical team members; } \\
\text { Training of surgical teams in IPC and AMS } \\
\text { (including nursing and pharmacy surgical } \\
\text { staff). }\end{array}$ & $\begin{array}{l}\text { Policy and Guidelines } \\
\text { Responsive to local needs and resource } \\
\text { limitations; } \\
\text { Co-developed with surgical, nursing, AMS, } \\
\text { IPC, ICU and anaesthesiology, patient } \\
\text { groups; } \\
\text { Active engagement and promotion of } \\
\text { surgical teams with policies and guidelines. }\end{array}$ \\
\hline $\begin{array}{l}\quad \text { Interdisciplinary Teamwork } \\
\text { Co-management of patients across } \\
\text { professional boundaries from admission to } \\
\text { discharge and follow-on community care; } \\
\text { Nursing and pharmacy involvement in } \\
\text { surgical rounds; } \\
\text { Recognising patient role as active } \\
\text { participant in their own care, particularly } \\
\text { post-operatively. }\end{array}$ & $\begin{array}{l}\text { Measuring Change in Outcomes } \\
\text { Prioritising monitoring of infection related } \\
\text { outcomes in surgical patients (including } \\
\text { SSIs and HCAI); } \\
\text { Developing a mechanism for effective, } \\
\text { relevant, and timely feedback of } \\
\text { behaviours to measure change e.g. HCAI } \\
\text { care bundles; hand hygiene; linking } \\
\text { infection outcomes to length of stay. }\end{array}$ \\
\hline & \\
\hline
\end{tabular}


Figure 4

A framework for more integrated care in infection management across surgical pathways

\section{Supplementary Files}

This is a list of supplementary files associated with this preprint. Click to download.

- SinghetalSupplementaryMaterialv2.docx 